\title{
A Novel Approach for Color Image Feature Extraction using Swarm Intelligence
}

\author{
A. D. Joshi \\ PG Student \\ Late G. N. Sapkal College of Engineering, Nashik \\ Savitribai Phule Pune University
}

\begin{abstract}
This paper reports research into representing edge detectors, with the help of swarm intelligence [1]. Other gradient-based detectors may not produce the same edge pixels if applied onto the same image. Hence, the need of an edge detector arises, which is precise in detecting edges in majority of the common types of edges. The idea behind Swarm intelligence arises from the insects, bird flocks, fish schools, and wildebeest herds etc. They all have some common features, they move in groups which are having a special behavior. Their coordination is so good and it observed that as some centralized controller dictates all movement. With this background, swarm intelligence relates with ants which may not be very clever individually, but ant colonies are capable of searching, making plans, and optimizing routes to food. Ant colonies are so good at finding the shortest path from one location to another, that an algorithm was developed based on their behavior called as Ant Colony Optimization. Keeping this idea in mind the paper extends previous work of an ant algorithm which is used for edge detection of gray image. Here, ACO is applied for feature extraction and edge pattern detection of a color image. An adaptive threshold histogram method is introduced to the ACO. An image is distributed separately as a combination of three primary colors $\operatorname{Red}(\mathrm{R})$, Green (G) and Blue (B). Based on these color intensities three separate histograms are generated and their average mean value is computed which is the threshold value. Thus, a threshold value is generated dynamically every time.
\end{abstract}

\section{General Terms}

Edge Detection, Feature Extraction, Swarm Intelligence, pheromone updation and evaporation

\section{Keywords}

Adaptive Threshold, Ant Colony Optimization, Histogram.

\section{INTRODUCTION}

Swarm intelligence is relatively a new technique used for problem-solving. It is motivated by the social behaviors of insects and of other animals. Particularly, number of methods and techniques inspired by ants and in between them the most studied and the most successful general purpose optimization technique is called as ant colony optimization. Ant colony optimization (ACO) is inspired by their division of labor and foraging behavior of some ant species. The individuals are often inefficient to perform the labor they might perform, but in group they might consistently work as fighters, scouts, nurses, undertakers, foragers, or cleaners with a defined line of work in a factory [1]. A chemical substance known as the pheromone is deposited by these ants on the ground to mark some promising path that should be followed by other members of the colony. A pheromone is used to improve the probability of other ants choosing the specified path as more pheromone is left when more ants go through the trip. The chemical trail i.e. pheromone has a falling action over time

\author{
J. V. Shinde \\ Faculty \\ Late G. N. Sapkal College of Engineering, Nashik \\ Savitribai Phule Pune University
}

due to the evaporation of trail and the quantity left by ants depends on the number of ants using this trail. There is an indirect communication between the ants by way of chemical pheromone trails that enables them to find shorter paths between their nest and food sources. This real ant colonies characteristic is used in ACO algorithms to solve, discrete optimization problems [2]-[4].

ACO is primarily used for detection of edges in a grayscale images. The templates are used to study self organization process, where a template can be defined as a pattern used to construct another pattern [5]-[6]. The ACO uses distributed nature of swarm intelligence methods that allow us to utilize such nature inspired approaches to increase efficiency in the self organizing process and to improve the performance of problem solving.

Image segmentation is an important feature of image processing where image is divided into different regions [7].To achieve this different edge detection methods have been introduced.

In this paper $\mathrm{ACO}$ is presented to be used for detecting edges in color image as edge detection in color image is an important feature. The limitations occur due to static threshold value generation in case of ACO for gray scale image feature extractions are eliminated as the threshold value is generated dynamically. By giving the facility of dynamically adjusting threshold parameters to individual swarm agents in response to the changing environment, increases the self-organization capability of Swarm by increasing adaptability. Because of this the Swarm allows to successfully self-organize into patterns representing the image feature structure over a wider range of image characteristics (such as ambient lighting, entropy, noise) [8].

The paper is organized as follows. Section2 outlines the origins of ACO algorithms. Particularly, the foraging behavior of real ant colonies is presented and how this behavior can be transferred into a technical algorithm for discrete optimization is shown. In Section 3 a description of the ACO for image feature extraction of the color image is provided. In Section 4, some important results are discussed. In Section5, conclusion is offered.

\section{LITERATURE SURVEY}

The Previous work on image edge detection performed by various researchers is given below.

Raman Maini \& Dr. Himanshu Aggarwal proposed a variety of image edge detection techniques of Gradient and Laplacian based edge detection and their comparison. Gradient-based algorithms such as Sobel and Prewitt filter have a major drawback as they are very sensitive to noise. The kernel size and coefficients are fixed and cannot be adapted to a given image. To overcome this, an adaptive edge detection algorithm is used to provide a robust solution which is adaptable to the varying noise levels of these images to help 
distinguish valid image contents from visual artifacts introduced by noise.

Bonabeau, B., Dorigo, M., \&Theraulaz, G. propose that every single insect in a social insect colony seems to have its own plan, and so far an insect colony looks so organized. No supervisor is needed for the seamless integration of all individual activities. What is the basic idea underlying all antbased algorithms? It is to use a positive feedback mechanism, based on an analogy with the trail-laying trail following behavior of some species of ants and some other social insects, to reinforce those portions of good solutions that contribute to the quality of these solutions, or to directly reinforce good solutions [9].

The foraging behavior of ants has inspired a novel approach to distributed optimization, Ant Colony Optimization (ACO).A virtual pheromone, used as reinforcement, allows good solutions to be kept in memory, from where they can be used to make up better solutions. ACO algorithm is now applied to many different combinatorial optimization problems ranging from the quadratic assignment and the sequential ordering problems to vehicle routing and graph coloring problems [10][12].

The artificial swarm-intelligent systems are expected to exhibit the features that may have made social insects so successful in the biosphere: flexibility, robustness, decentralized control, and self-organization. There are some issues, to the application of swarm intelligence to solve problems:

1) First, it would be very useful to define methodologies to "program" a swarm or multiagent system so that it performs a given task.

2) Second, and perhaps even more fundamental than the issue of programming the system, is that of defining it: How complex should individual agents be?

Rob J. Mullen, Dorothy N. Monekosso, Paolo Remagnino, propose that so far only image edge gradient is considered as the heuristic information that is used to guide the ant agents. The effects of self-organization and pattern formation using different heuristic information are used to guide the ant agents for alternative image features.

The images used in ACO have been grayscale previously. Here, RGB color images are used, and the heuristic information used to guide the ants towards areas of the image with a prominent intensity of a particular color which can be changed.

Shweta Agarwal performed Edge detection in Blurred images using Ant Colony Optimization Technique. Edge detection in blurred digital images and prioritized them using different color values according to their strength and importance. Here algorithm does not consider image deblurring hence eliminating any chances of data loss and a blur image will produce multiple edges in an area of concern is few among these edges will be non-prominent and less useful.

Anna Veronica Baerina, Carlos Oppus proposed edge detection using ant colony optimization in which they established a pheromone matrix that represents the edge information at each pixel based on the routes formed by the ants dispatched on the image.

Here results are based on at different values of parameter controlling the degree of exploration of the ants. Increase in the parameter value results in smoother edges. The value should be between, but not equal to, 0 and 1 because it causes some significant features to be missed. Therefore, higher values of parameter controlling the degree of exploration of the ants are suitable for images that contain less amount details while lower values are suitable for those that contain more details.

Edges in gray-level images are pixel locations of abrupt graylevel change. In a gradient based methods a change in the image function can be described by a gradient which points in the direction of the largest growth of the image function. This method works best in case of abrupt gray-level transition, like a step function. For the wider transition region, it is advantageous to apply second-order derivatives like the Laplacian and the potential edge pixel locations can be described by zero-crossings in the results.

Edge detection in gray-level images is a well-established area, but edge detection in color images has not received the same attention. The primary difference between color images and gray-level images is that, in a color image, a color vector (which generally having three components Red, Green and Blue) is assigned to a pixel, while a scalar gray-level is assigned to a pixel of a gray-level image [19]- [21].

\section{ACO FOR GRAYSCALE IMAGE}

This algorithm is based on the workings of original AS (Dorigo \& Gambardella, 1997), with an updated, application specific pheromone update rule and heuristic information.

The algorithm uses artificial ants as a simple computational agent. It is initialized with $\mathrm{N}$ ants that occupy 'random' pixels within the image; here the image pixels are equivalent to states in the search environment. The aim of the ants is to locate and plot the boundaries within the image. To achieve this heuristic information is introduced. With each move to a new pixel every ant deposits an amount of pheromone, where the amount deposited may be a function of, e.g. change in image gradient, and pheromone evaporation occurs at a fixed rate per iteration. The transition rule is then a function of heuristic information and pheromone map [13].

\subsection{Initialization Phase}

Every image I size is represented by $\mathrm{M}$ x N. Fig. 1 shows a two dimensional graph representation of an Image. The ' $\mathrm{K}$ ' specifies number of artificial ants. These ' $\mathrm{K}$ ' ants are taken as input and randomly distributed over the entire image such that every pixel is considered as a node. [19]

\subsection{Construction Phase}

At each time step $\mathrm{t}$, one ant among $\mathrm{N}$ ants is randomly selected which moves a distance of 1 pixel to one of the eight surrounding pixels. The movement of ant is shown in fig. 1 .

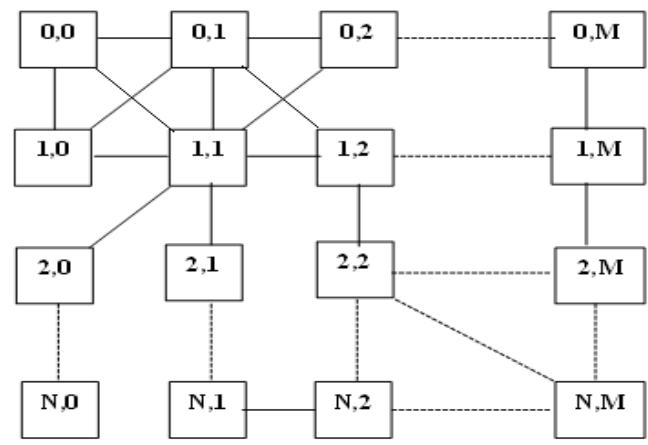

Fig 1: Graph Representation of M X N 2 D Image 
$P_{(l, m)}^{(n)} T_{(i, j)}^{(n-1)}=\frac{\left(\tau_{i, j}^{n-1}\right)^{\alpha}\left(\eta_{i, j}^{\beta}\right)}{\sum_{l i, j) \in \Omega_{(l, m)}}\left(\tau_{(i, j)}^{n-1}\right)^{\alpha}\left(\eta_{i, j}^{\beta}\right)}$

In the above equation ' $\tau$ ' represents the pheromone value at node ( $\mathrm{i}, \mathrm{j}), \Omega_{(1, \mathrm{~m})}$ is the neighbouring node of node $(1, \mathrm{~m})$. The heuristic information at node $(i, j)$ is represented by $\eta_{i, j}$. The influence of the pheromone matrix and heuristic matrix is shown by the constants $\alpha$ and $\beta$ respectively.

\begin{tabular}{|c|c|c|c|c|}
\hline$I_{i-2, j-2}$ & $I_{i-2, j-1}$ & $I_{i-2, j}$ & $I_{i-2, j+1}$ & $I_{i-2, j+2}$ \\
\hline$I_{i-1, j-2}$ & $I_{i-1, j-1}$ & $I_{i-1, j}$ & $I_{i-1, j+1}$ & $I_{i-1, j+2}$ \\
\hline$I_{i, j-2}$ & $I_{i, j-1}$ & $I_{i, j}$ & $I_{i, j+1}$ & $I_{i, j-2}$ \\
\hline$I_{i-1, j-2}$ & $I_{i+1, j-1}$ & $I_{i-1, j}$ & $I_{i-1, j+1}$ & $I_{i+1, j+2}$ \\
\hline$I_{i-1, j-2}$ & $I_{i-2, j-1}$ & $I_{i-2, j}$ & $I_{i+2, j-1}$ & $I_{i+2, j+2}$ \\
\hline
\end{tabular}

Fig 2: Clique Matrix

The process has important issues as shown below:

1) Heuristic information: It can be determined by local statistics of the image. The local statistics depends on clique $\mathrm{c}$. The local statistics at pixel location $(i, j)$ is calculated by following formula [14]:

$\eta_{i, j}=\frac{1}{Z\left(V_{c}\left(I_{i, j}\right)\right)}$

Here, $\mathrm{Z}$ is normalization factor, it denotes the function of a local group of pixels ' $c$ ' that is called as clique [15].

$Z=\sum_{i=1: M 1} \sum_{j=1: M 2} V_{c}\left(I_{i, j}\right)$

The value of the pixel $(i, j)$ is depends on variation of intensity values in the clique $\mathrm{c}$. The visibility at each pixel is calculated by following eight equations:

$$
\begin{aligned}
& \eta_{x-1, y-1}=|(I(x-1, y)-\mathrm{I}(\mathrm{x}, \mathrm{y}-1))| \\
& \eta_{x-1, y}=|(I(x-1, y-1)-\mathrm{I}(\mathrm{x}-1, \mathrm{y}+1))| \\
& \eta_{x-1, y+1}=|(I(x-1, y)-\mathrm{I}(\mathrm{x}, \mathrm{y}+1))| \\
& \eta_{x, y-1}=|(I(x+1, y-1)-\mathrm{I}(\mathrm{x}-1, \mathrm{y}-1))|(7) \\
& \eta_{x, y+1}=|(I(x-1, y+1)-\mathrm{I}(\mathrm{x}+1, \mathrm{y}+1))| \\
& \eta_{x+1, y-1}=|(I(x, y-1)-\mathrm{I}(\mathrm{x}+1, \mathrm{y}))| \\
& \eta_{x+1, y}=|(I(x+1, y-1)-\mathrm{I}(\mathrm{x}+1, \mathrm{y}+1))|(10) \\
& \eta_{x+1, y+1}=|(I(x+1, y)-\mathrm{I}(\mathrm{x}, \mathrm{y}+1))|
\end{aligned}
$$

The function $\mathrm{V}_{\mathrm{c}}\left(\mathrm{I}_{\mathrm{i}, \mathrm{j}}\right)$ is determined by following equation.

$$
\begin{gathered}
V_{c}\left(I_{i, j}\right)=f\left(\left|I_{i-2, j-1}-I_{i+2, j+1}\right|\right. \\
+\left|I_{i-2, j+1}-I_{i+2, j-1}\right| \\
+\left|I_{i-1, j-2}-I_{i+1, j+2}\right|+\left|I_{i-1, j-1}-I_{i+1, j+1}\right| \\
+\left|I_{i-1, j}-I_{i+1, j}\right|+\left|I_{i-1, j+1}-I_{i-1, j-1}\right| \\
\left.+\left|I_{i-1, j+2}-I_{i-1, j-2}\right|+\left|I_{i, j-1}-I_{i, j+1}\right|\right)
\end{gathered}
$$

The function $f(x)$ is determined by the following equations given below:

$$
\begin{aligned}
& f(x)=\lambda_{x}, \text { for } x \geq 0 \\
& f(x)=\lambda_{x 2} \text {, for } x \geq 0 \\
& f(x)=\left\{\begin{array}{lr}
\sin \left[\frac{\pi x}{2 \lambda}\right], 0 \leq x \leq \lambda \\
0, \quad \text { else }
\end{array}\right. \\
& f(x)=\left\{\begin{array}{lr}
\sin \left[\frac{\pi x}{2 \lambda}\right], & 0 \leq x \leq \lambda \\
0, & \text { else }
\end{array}\right.
\end{aligned}
$$

The function can be determined by slightly changing the value of $\lambda$.

\section{2) Permissible range of ant movements:}

It is nothing but the. $\Omega(1, \mathrm{~m}))$ in the position $(1, \mathrm{~m})$. Here, $\mathrm{I}(\mathrm{x}$, $y)$ is image intensity at each pixel location $(\mathrm{x}, \mathrm{y})$ and $\eta \mathrm{ij}$ depends on direction of ant movement.

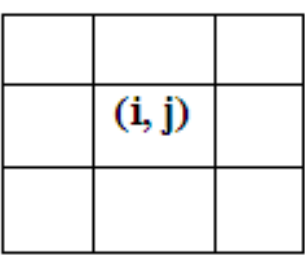

(A)

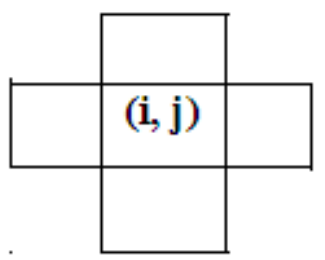

(B)
Fig 3 (A): node (i, j) has 8 Connectivity Neighborhood [11], (B) node (i, j) has 4 Connective Neighborhood

The ants at any position $(1, \mathrm{~m})$ can walk in either any of the 4 directions $(\mathrm{E}, \mathrm{W}, \mathrm{N}, \mathrm{S})$ or in 8 directions $(\mathrm{NW}, \mathrm{N}, \mathrm{NE}, \mathrm{W}, \mathrm{E}$, $\mathrm{SW}, \mathrm{S}, \mathrm{SE})$ as shown in the Figure 3.

One more parameter that needs attention is the Ant's memory length. The ant's memory locations are nonadmissible so, its choice is a crucial one. Small length may cause the algorithm idle whereas large length might miss the details. It is empirically chosen [15] in the interval [0.85 A, $1.15 \mathrm{~A}]$ where A is 40 for image of size $128 \times 128$.

\subsection{Update Phase}

In this process the pheromone matrix is updated after the two update operations. The first update takes place after movement of each ant in each construction-step. Each building block of pheromone matrix is modified as given in equation (14) and the second update is performed after movement of entire ants in all construction-step as given in equation (15).

$\tau_{i, j}^{n-1}$

$=\left\{\begin{array}{cc}(1-\rho) * \tau_{i, j}^{n-1}+\rho * \Delta_{i, j}^{k}, & \text { if }(i, j) \text { belongs to best tour } \\ \tau_{i, j}^{n-1} & , \quad \text { Otherwise }\end{array}\right.$

$\tau_{i, j}^{n}=(1-\Psi) \tau_{i, j}^{n}+\Psi * \tau_{0}$

In this $\psi$ is the pheromone decay coefficient. The search for subsequent ants is broadened by local update which reduced the pheromone level on the traversed edges. It provides an opportunity for the subsequent ants to produce necessary solutions and hence, the chance of repetition becomes less likely in the same iteration [16]. 


\subsection{Decision Phase}

In this step, the solution is established from the values present in final pheromone matrix. To determine whether it is edge or not a binary decision is made at each pixel location. By applying the threshold value $\tau$ on final pheromone matrix the decision is made.

\subsection{Visualization Phase}

Here, different values of the $\mathrm{Si}(\psi)$ parameter are applied to the above algorithm. If the value of the phi parameter is smaller the algorithm detects more edges in the image. By decreasing the value of the phi parameter, output of the given image becomes clearer [17] but the value should not be zero.

\section{PROPOSED METHODOLOGY}

The proposed color image edge detection based on ACO is applied on a 2D image for generation of a pheromone matrix. Each entry of that pheromone matrix represents the intensity change in the original image influenced by the edge location. The adaptive threshold value is generated at every time which is used in Decision Process by ants and avoids over segmentation of image.

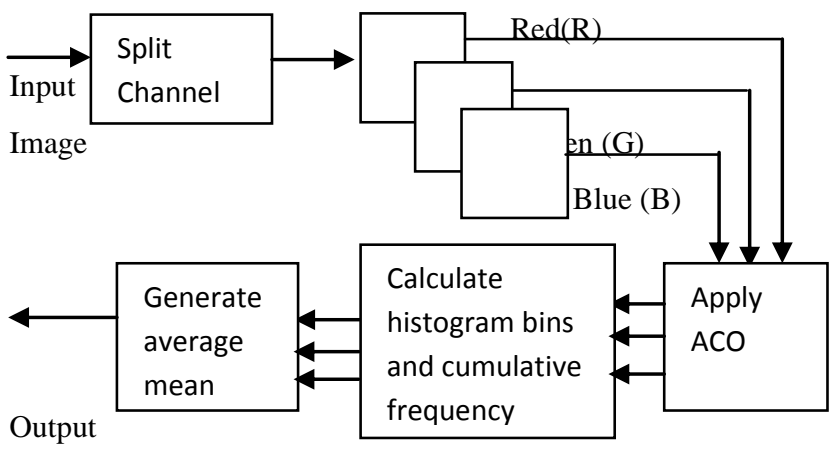

Image

Fig 4: Block Diagram of Proposed System

The ACO is implemented for extracting RGB feature extraction. The heuristic information is used to guide the ants towards areas of the image with a prominent intensity of a particular color which can be changed. The Adaptive threshold Histogram technique is used. It splits up image into three primary colors. As per the color intensity values present in an image three separate histograms are generated for each image. Then cumulative frequencies are calculated and average mean value is generated. Then finally the result is fused [18]. These values depend on the input color image.

\section{EXPERIMENTAL RESULTS}

Experiments are conducted to evaluate the performance of the proposed approach on the basis of peak signal to noise ratio shown in Table 1 using some experiment images, five Quercus Family Leaf images, Lena, Church, Elephant, House, Flight are shown in Figure 5-9 and Figure 10-16. All these images are taken from the dataset "Berkely Segmentation Dataset:http://www.eecs.berkeley.edu/Research/Projects/CS/v ision/bsds/ “"

\subsection{Comparing PSNR of Different Algorithms}

Figures 5-13 below shows the performance of proposed algorithm with Sobel, Canny and Prewitt algorithms and Table 1 shows performance of proposed method w.r.t other existing algorithms by comparing their PSNR values on the experimental images mentioned above.

a) Result on Leaf Images

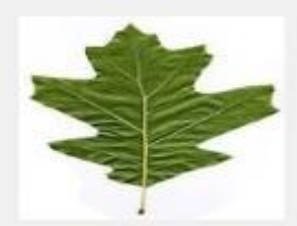

Leaf1

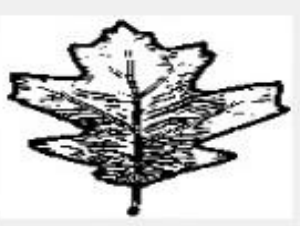

(a)

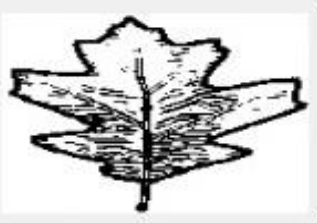

(b)

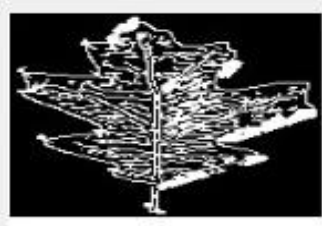

(c)

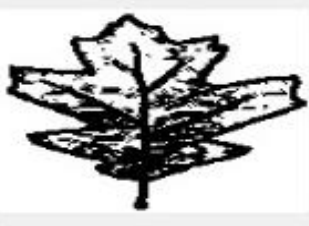

(d)

Fig 5: Leaf1: (a) Sobel Edges, (b) Prewitt Edges, (c) Canny Edges, (d) Proposed Method

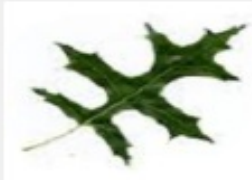

Leaf2

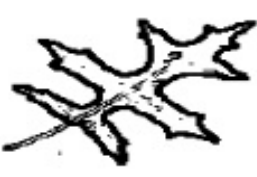

(a)

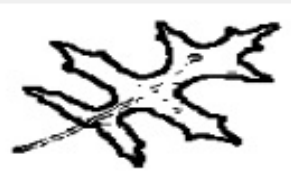

(b)

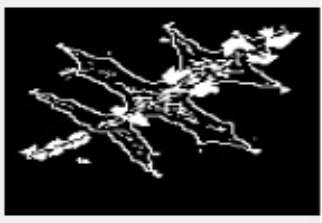

(c)

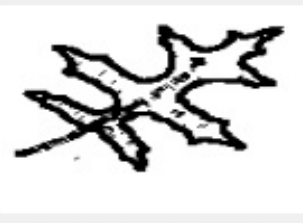

(d)

Fig 6: Leaf 2:(a) Sobel Edges, (b) Prewitt Edges, (c) Canny Edges, (d) Proposed Method

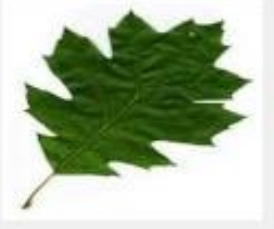

Leaf3

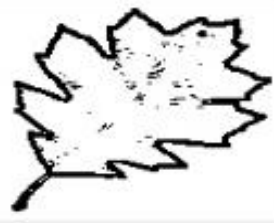

(a)

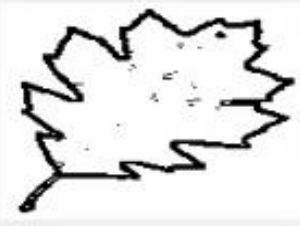

(b)

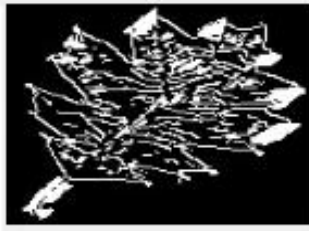

(c)

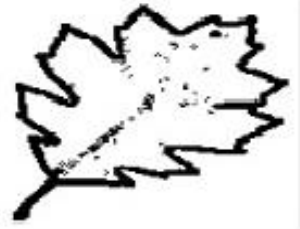

(d)

Fig 7: Leaf3: (a) Sobel Edges, (b) Prewitt Edges, (c) Canny Edges, (d) Proposed Method 

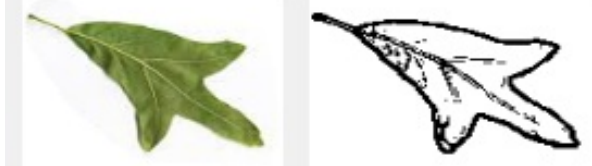

Leaf4

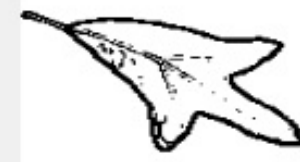

(b)

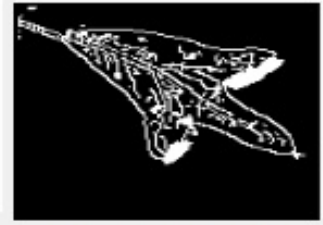

(c)

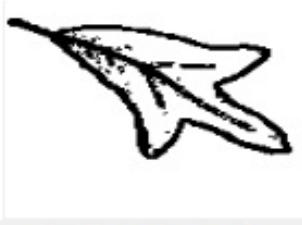

(d)

Fig 8: Leaf4: (a) Sobel Edges, (b) Prewitt Edges, (c) Canny Edges, (d) Proposed Method

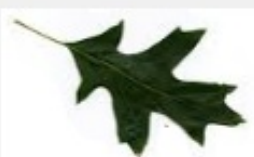

Leaf5

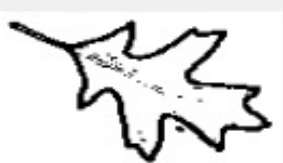

(a)

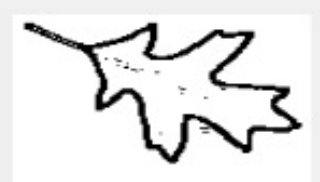

(b)
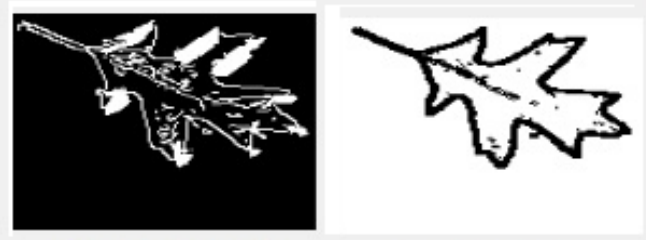

(c)

(d)

Fig 9: Leaf5: (a) Sobel Edges, (b) Prewitt Edges, (c) Canny Edges, (d) Proposed Method

b) Result on Other than Leaf Images

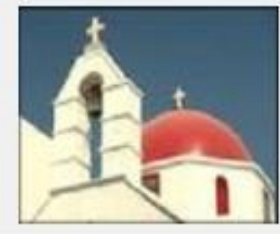

Church

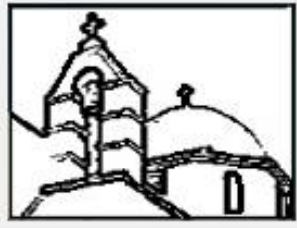

(a)

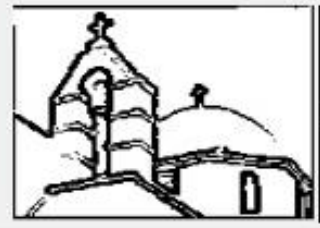

(b)

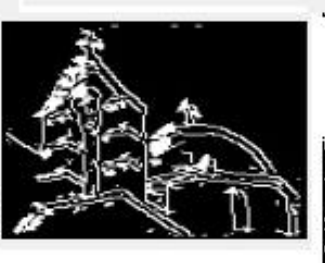

(c)

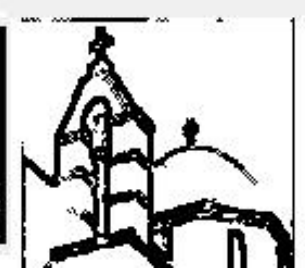

(d)

Fig 10: Church: (a) Sobel Edges, (b) Prewitt Edges, (c) Canny Edges, (d) Proposed Method

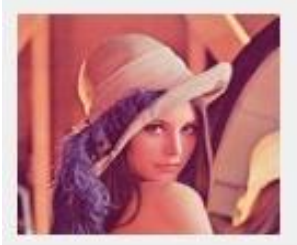

Lena

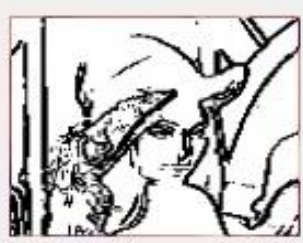

(a)

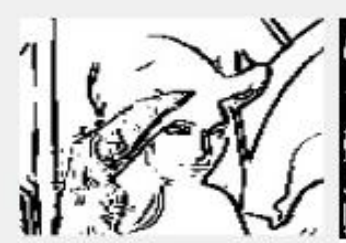

(b)

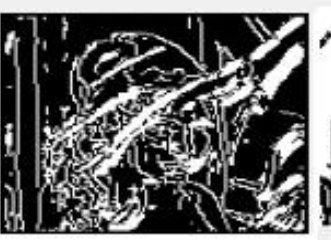

(c)

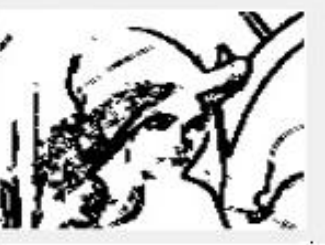

(d)

Fig 11: Lena: (a) Sobel Edges, (b) Prewitt Edges, (c) Canny Edges, (d) Proposed Method

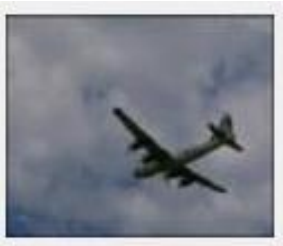

Flight

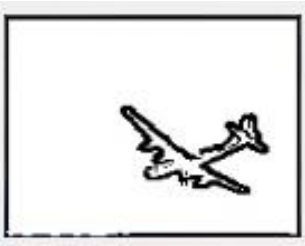

(a)

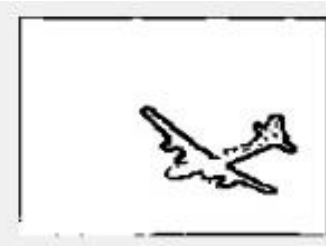

(b)

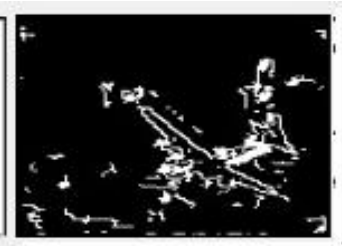

(c)

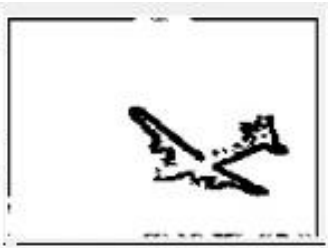

(d)

Fig 12: Flight: (a) Sobel Edges, (b) Prewitt Edges, (c) Canny Edges, (d) Proposed Method

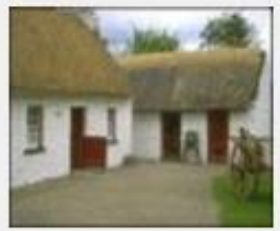

House

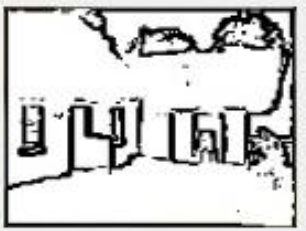

(a)

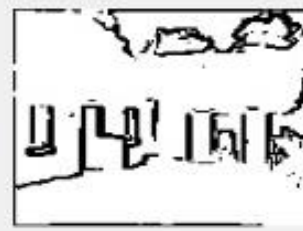

(b)

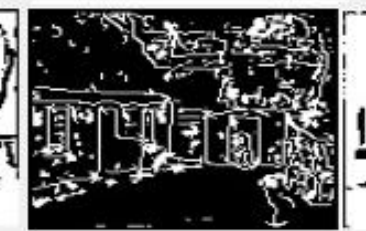

(c)

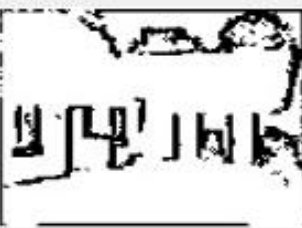

(d)

Fig 13: House: (a) Sobel Edges, (b) Prewitt Edges, (c) Canny Edges, (d) Proposed Method 


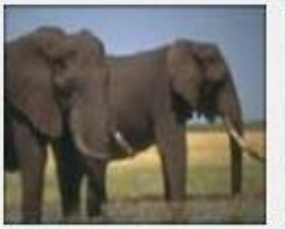

Elephant

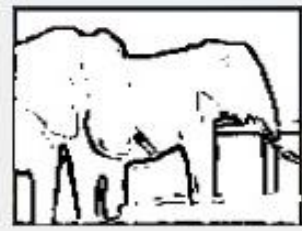

(a)

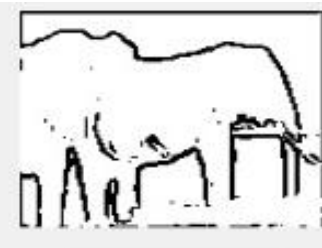

(b)

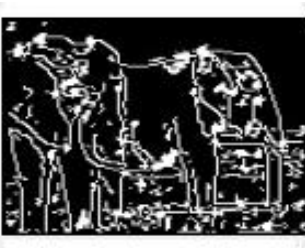

(c)

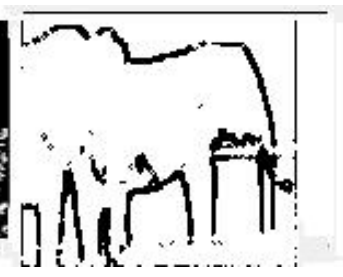

(d)

Fig 14: Elephant: (a) Sobel Edges, (b) Prewitt Edges, (c) Canny Edges, (d) Proposed Method

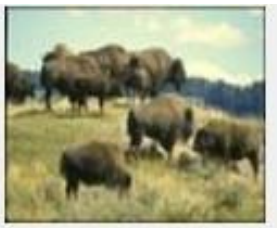

Animals

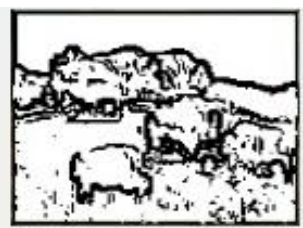

(a)

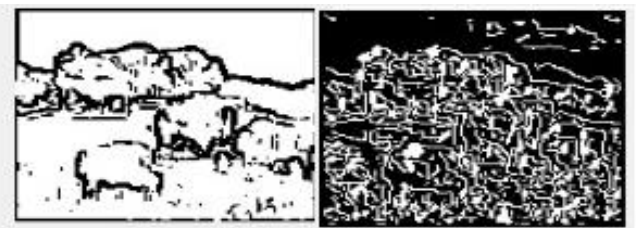

(b)

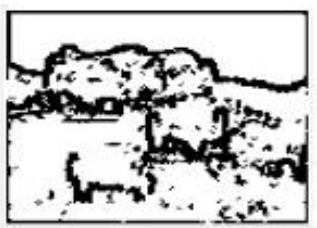

(d)

Fig 15: Animals: (a) Sobel Edges, (b) Prewitt Edges, (c) Canny Edges, (d) Proposed Method

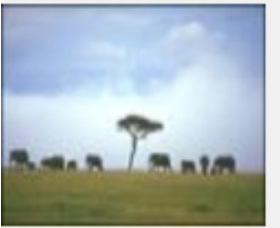

Animals \& Tree

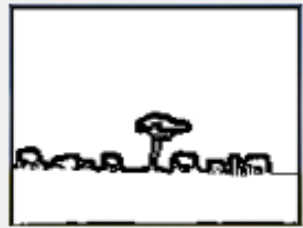

(a)

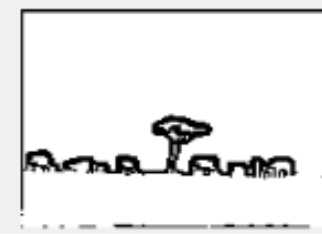

(b)

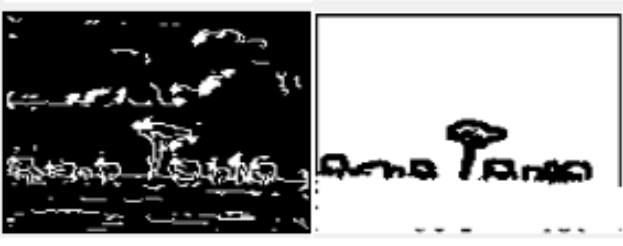

(c)

(d)

Fig 16: Animals and Tree : (a) Sobel Edges, (b) Prewitt Edges, (c) Canny Edges, (d) Proposed Method

Table 1: The PSNR Values

\begin{tabular}{|c|c|c|c|c|c|}
\hline $\begin{array}{c}\text { Sr. } \\
\text { No. }\end{array}$ & Image & $\begin{array}{c}\text { PSNR } \\
\text { Sobel }\end{array}$ & $\begin{array}{c}\text { PSNR } \\
\text { Prewitt }\end{array}$ & $\begin{array}{c}\text { PSNR } \\
\text { Canny }\end{array}$ & $\begin{array}{c}\text { PSNR } \\
\text { Proposed } \\
\text { System }\end{array}$ \\
\hline 1. & Leaf1 & 6.81 & 5.89 & 1.68 & 7.83 \\
\hline 2. & Leaf2 & 8.68 & 7.68 & 0.93 & 9.15 \\
\hline 3. & Leaf3 & 4.38 & 3.96 & 1.98 & 4.89 \\
\hline 4. & Leaf4 & 8.86 & 7.78 & 0.98 & 9.52 \\
\hline 5. & Leaf5 & 7.65 & 6.86 & 1.01 & 8.34 \\
\hline 6. & Church & 4.76 & 4.80 & 4.26 & 4.79 \\
\hline 7. & Lena & 4.68 & 4.45 & 3.56 & 5.22 \\
\hline 8. & Flight & 5.51 & 5.40 & 5.65 & 5.72 \\
\hline 9. & House & 4.30 & 4.20 & 5.30 & 4.52 \\
\hline 10. & Elephant & 3.93 & 3.80 & 6.26 & 4.18 \\
\hline 11. & Animals & 5.37 & 5.07 & 4.12 & 5.49 \\
\hline 12. & Tree \& & 7.53 & 7.32 & 2.50 & 7.48 \\
& Animals & & & & \\
\hline
\end{tabular}

Fig 17-18 shows comparison of ACO with other techniques based on PSNR values. Fig 17 shows PSNR Values of Leaf Images and Fig 18 show PSNR Values of non leaf images.

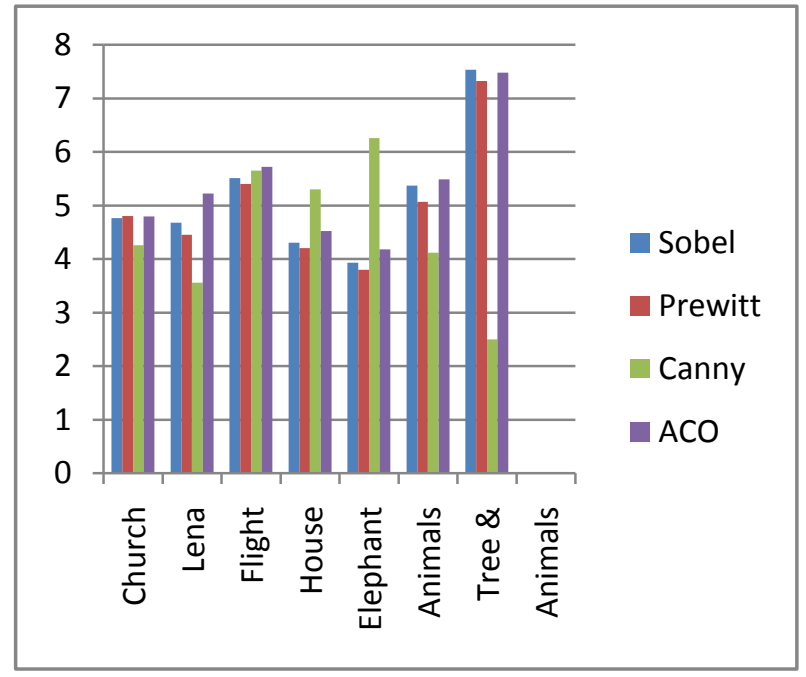

Fig 17: Comparison with Other Techniques based on PSNR Values of Non-Leaf Images

\section{CONCLUSION}

A new approach to the ant colony optimization method have been implemented and tested in color image feature extraction. The Experiments shows the superiority of the new method as compared to previous specially in selecting static threshold value which results in oversegmentation of image. The threshold value is generated dynamically depending on the image every time by using Adaptive Threshold Histogram method. The dynamic selection of threshold value results in avoiding oversegmentation of image. Comparisons with wellknown existing methods have been made and comparative results are generated with respect to PSNR values on sample dataset of leaf images and non-leaf images. The analysis has 
noted some limitations such as image size which is typically of size $128 \times 128$.

The future enhancement of an algorithm is to create multiple swarms such that their working is independent of each other. One swarm may use to detect background edges of image and other may use to detect internal parts of image. These results in lesser time for implementation of algorithm and simultaneously more than one features from image can be detected.

\section{REFERENCES}

[1] Fernandes, C., Ramos, V., \& Rosa A.,"Varying the population size of artificial foraging swarms on time varying landscapes In W. Duch, J. Kacprzyk, E. Oja, \& S. Zadrozny (Eds.), Artificial neural networks: Biological inspirations - ICANN 2005. Lecture notes in computer science", Berlin/Heidelberg: Springer, Vol. 3696, pp. $311-316$.

[2] Bonabeau, B., Dorigo, M., \& Theraulaz, G., "Ant Foraging Behaviour", in Swarm intelligence from natural to artificial systems. New York, NY: Oxford University Press, 1999, pp. 25-65.

[3] Marco Dorigo and Thomas Stützle, "Ant Colony Optimization Theory", in Ant Colony Optimization, Cambridge, Massachusetts London, England, MIT Press, pp. 121-151.

[4] Marco Dorigo, Mauro Birattari, and Thomas St"utzle, "Ant Colony Optimization Artificial Ants as a Computational Intelligence Technique", IEEE Computational Intelligence Magazine “, pp. 28-39, November-2006.

[5] Fernandes, C., Ramos, V., \& Rosa, A. C.," Self-regulated artificial ant colonies on digital image habitats", International Journal of Lateral Computing, 2 (1), 1-8.

[6] Chittka, L., \& Muller, H., "Learning, specialization, efficiency and task allocation in social insects", Communicative and Integrative Biology, 2 (2), 151-154.

[7] Ramandeep Kaur1 and Balwinder Singh2, "Design and development of sapling monitoring system", International Journal of Computer Science, Engineering and Applications (IJCSEA) Vol.3, No.5, pp. 39-44, October-2013

[8] Shweta Agrawal, "A review paper of edge detection using Ant Colony Optimization Techniques", International Journal of Latest Research in Science and Technology ISSN (Online):2278-5299, PP. 120-124.

[9] Raman Maini \& Dr. Himanshu Aggarwal, "Study and Comparison of Various Image Edge Detection Techniques", International Journal of Image Processing (IJIP), Volume (3) : Issue (1), pp. 1-12.
[10] Rebika Rai, Ratika Pradhan , M.K.Ghose, "Ant based Swarm Computing Techniques for Edge Detection of Images- A Brief Survey", ISSN 2250-2459, ISO 9001:2008 Certified Journal, Volume 3, Issue 4, April 2013.

[11] Canny, J. A. (1986). Computational approach to edge detection. IEEE Transactions on Pattern Analysis and Machine Intelligence, 8, 679-698.

[12] J. Tian, W. Yu, and S. Xie, "An ant colony optimization algorithm for image edge detection," in IEEE World Congress on Evolutionary Computation, pp. $751-756$, Jun. 2008.

[13] Jing Tian, Weiyu Yu, and Shengli Xie, "An Ant Colony Optimization Algorithm for Image Edge Detection", IEEE Transactions, pp. 751-756, March 2009.

[14] Om Prakash Verma et. al., "A Novel Fuzzy Ant System for Edge Detection", in Proc. of the 9th IEEE International Conference on Computer and Information Science, pp.228-233, 2010.

[15] P. Xiao, J. Li, and J.-P. Li, "An improved ant colony optimization algorithm for image extracting," in Apperceiving Computing and Intelligence Analysis (ICACIA), 2010 International Conference on, pp. 248 252, Dec. 2010

[16] Anna Veronica Baterina and Carlos Oppus, "Image Edge Detection Using Ant Colony Optimization", International Journal of circuits, System and Signal Processing, Issue 2 vol.4, pp. 25-33, 2010.

[17] Ioannis M. Stephanakis and George C. Anastassopoulos, "Segmentation Using Adaptive Thresholding of the Image Histogram according to the Incremental Rates of the Segment Likelihood Functions, "unpublished", pp. 15.

[18] Charu Gupta and Sunanda Gupta, "Edge Detection of an Image based on Ant Colony Optimization Technique", International Journal of Science and Research (IJSR), India Online ISSN: 2319-7064, Vol. 2, No. 6, June2013, pp. 114-120.

[19] A. Koschan and M. Abidi, "Detection and Classification of Edges in Color Images," Signal Processing Magazine, Special Issue on Color Image Processing, Vol. 22, No. 1, 2005, pp. 64-73.

[20] Hasan Ihsan Turhan, Gozde Sahin and Aydan M. Erkmen," Comparing Color Edge detection Techniques"," unpublished", pp. 1-6.

[21] Rob J. Mullen, Dorothy N. Monekosso, Paolo Remagnino," Ant algorithm for image feature extraction", Expert Systems with Applications, 40 (2013) $4315-4332$ 